\title{
Discussion on Some Problems of electro-osmotic consolidation of soil
}

\author{
YE Zi-Jian \\ BGI Engineering Consultants Ltd., Beijing 100038, China
}

\begin{abstract}
Keywords: Electro-osmotic consolidation; Polarity reversal; Drainage consolidation; efficiency of electro-osmosis
\end{abstract}

Abstract. Although many laboratory tests study on electro-osmotic consolidation of soil, it is not widely used in practical engineering, since influenced by existing problems of electrode erosion, low effiency, uneven consolidation, the effect of electro-osmotic treatment is not perfect. In this paper, several laboratory test methods to improve the efficiency of electro-osmotic consolidation are discussed, advantage and disadvantage of each method is analysis based on test results.

\section{Introduction}

In recent years, with the size of reclamation land continues expand, relying on traditional gravity drainage consolidation method in dealing with low permeability of saturated clay is ineffective and consumes long time. Since electro-osmotic consolidation is not related to the drainage rate and the size of the soil particle, it becomes one of promising method in many new approaches for soft soil improvement. But due to its imperfect reinforcement mechanism, this method needs more research and discussion.

Electro-osmotic consolidation is a method that electrical energy is utilized to reinforce soft soil foundation. In 1808, Reuss found that water molecule in soil could be separated from soil particle, the model of water flow has been established. In the 1930's Casagrande aware that electro-osmotic treatment plays an important role in improve the fine grained soil shear strength and stability, and applied the electroosmosis technology to soft soil reinforcement in the geotechnical engineering, after that a lot of research work focus on aspects of mechanism and application of electro-osmotic consolidation are involved by international scholars ${ }^{[1]}$. Practical application for electro-osmotic consolidation still exists many problems and defects. This paper mainly presents the discussion about different laboratory methods conducted to improve the efficiency of electro-osmotic consolidation of soil, advantage and disadvantage of each method is analysis according to test results.

\section{Improvement principle and applicability}

Improvement principle of electro-osmotic consolidation already been known by many people. As we know soil is solid-liquid-gas phase dispersion system. Due to soil particle is negative charge, water molecule in soil is positive charge, insert metal electrodes with direct current into soil. Under the action of the current, water molecules in the vicinity of the anode moves toward the cathode where the potential is lower, and the soil particles are gradually loss water and consolidate, and the water molecules are discharge in the vicinity of the cathode to form consolidation settlement. The electro-osmotic flow rate is proportional to the potential gradient, and can be expressed by the similar form of the Darcy's law as:

$$
v=\frac{k_{e} \cdot V}{L}
$$

Where $v$ is the velocity, $V$ is the potential, $L$ is the distance of anode and cathode, $k_{e}$ is the coefficient of electro-osmotic permeability.

Research study shows that the applicable soil for electro-osmotic consolidation, the $k_{e}$ should be in the ranges of $2 \sim 5 \times 10^{-5}$, and the soil with fine particles, large electro-osmotic coefficient, and medium hydraulic permeability ${ }^{[2]}$. Since the effect of traditional drainage consolidation is good at 
discharge free water, soil with higher coefficient of hydraulic permeability using traditional drainage consolidation method can achieve good results, the effect of using electro-osmosis to discharge weakly bound water is obvious, soil with higher hydraulic coefficient owns larger soil particles and smaller proportion of bound water, so for fine grained soil, and soil with higher electro-osmotic coefficient is more suitable for using electro-osmosis to consolidate.

\section{The major problem of using electro-osmotic consolidation of soil:}

Electro-osmosis is a process of variety factors interaction and mutual coupling with each other, It involves soil mechanics, electricity, chemistry, and many other disciplines, the domestic and foreign research on electro-osmotic consolidation is not perfect, especially for problems of electrode erosion, low electroosmosis efficiency, uneven consolidation. Such problems are still difficult to solve.

(1) Electrode erosion

In electro-osmotic consolidation method, metal electrode is commonly used as electrode, with the current gradually applied to each side of electrode, the cation of electrode has electrochemical reaction with water molecules in soil, metal electrode is easily destoried by corrosion, especially for the anode part where the conductive ability is seriously influenced.

(2) Low efficiency

According to study on application of electro-osmotic consolidation method in the field, An important amount of the electrical potential is indeed lost at the soil-electrode contact face and only $35 \%$ of the gradient are effectively applied to electrode ${ }^{[3]}$.

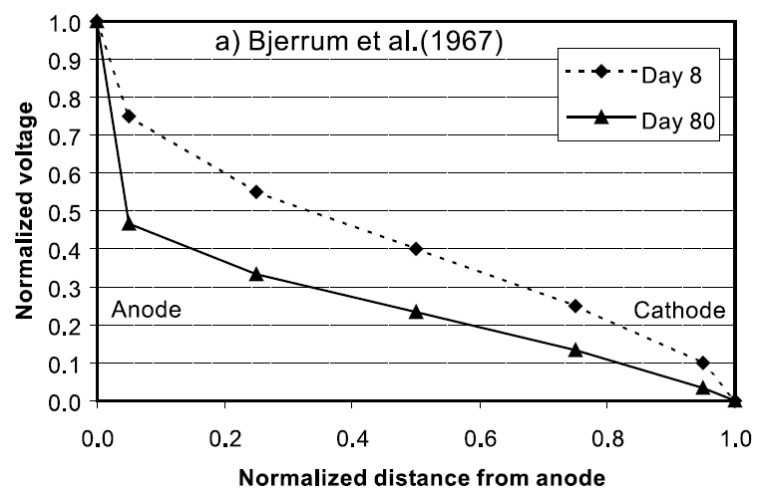

Fig.1 Potential loss during electro-osmotic consolidation

At the early stage of the test, due to the increase of resistivity, potential consumption near the anode becomes larger, drainage efficiency gradually reduced with the time of current applied. (See Fig.1)

(3) Uneven consolidation

Due to main water molecules moves toward the cathode during electro-osmosis, as Eq. 2 and Eq.3 shows that through electrolysis reaction, oxygen is produced near the anode, hydrogen is produced near the cathode. Chemical Reaction equation:

Anode: $2 \mathrm{H}_{2} \mathrm{O} \geq \mathrm{O}_{2}(\mathrm{~g})+4 \mathrm{H}^{+}+4 e^{-}$

Cathode: $4 \mathrm{H}_{2} \mathrm{O}+4 e^{-} \geq 2 \mathrm{H}_{2}(\mathrm{~g})+4 \mathrm{OH}^{-}$

With water molecules moves toward the cathode, water content near the cathode will not change too much, cause the degree of consolidation near anode is more than cathode, and makes soil uneven consolidation, the settlement at the anode is far greater than near the cathode, this phenomenon sometimes can make soil specimen cracking during test.

\section{Methods to improve the efficiency of electro-osmotic consolidation}

Through study on the problem of electro-osmotic consolidation, experimental methods such as polarity reversal, alternating current (AC) for soil improvement or insert saline solution has been established. EKG new material is developed for better overcome the defects, and electro-osmotic 
consolidation combine with traditional consolidation method is also significance for electro-osmosis application.

\section{Polarity reversal}

Due to non-homogeneity soil settlement during application of electro-osmotic consolidation, for the purpose of get even settlement, regular reverse two polarities in the process of insert current, cathode converts to anode, anode converts to cathode, so that the direction of water flow in soil changes with the polarity reversal, and soil settlement becomes even.

For example, the power during test use voltage of $1 \mathrm{v} / \mathrm{cm}$, polarity reversal cycle is 75 minutes, time for current insert anode and cathode is equal, based on analysis and observation of the undrained shear strength, water content, drainage volume of soil mass near the andoe and cathode, the test result is shown as below in Fig. $2^{[4]}$.
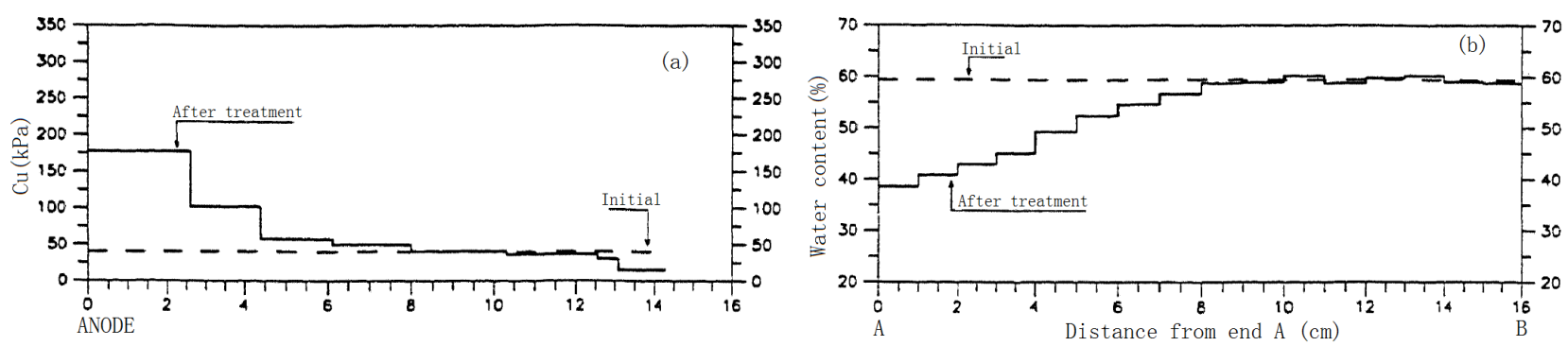

Fig.2 Distribution of undrained shear strength (a) and water content (b) after treatment

The experimental results shows that the polarity reversal treatment is different from usual electro-osmotic consolidation, water content of usual electro-osmotic consolidation near cathode during the experiment is changed alittle, the changes of water content are mainly concentrated in the vicinity of the anode. From Fig.4, in every 75 minutes exchange electrode position, made the soil water content distributed uniformly, water content near anode and cathode are $15 \%$ lower. During polarity reversal, the efficiency of drainage consolidation is directly proportional to the applied voltage. In the vicinity of anode, with the time of current load and water molecules gradually electrolysis, soil shear strength is increased, and PH value is gradually reduced with the increasing of hydrogen ion, and PI is increased. In the vicinity of cathode, soil shear strength changed very little, PH value as influenced by the electrolysis of water increase with hydroxyl ions, PI is gradually reduced.The effect of PH value changes with the electrochemical reaction.

Through the way of polarity reversal, water in soil between the anode and cathode moves back and forth, the experiment was able to obtain even settlement, soil consolidation near the cathode is greater than without polarity reversal treatment under the same condition. However, soil consolidation by polarity reversal also has some drawbacks.

(1) High engery consumption, low efficiency.

Drainage consolidation under the same condition to produce the same mount of settlement, energy consumption for polarity reversal treatment is greater than usual electroosmotic consolidation. Each consume $1 \%$ energy, drainage volume of usual electroosmotic consolidation is greater than polarity reversal treatment. This is largely due to reverse the electrode to force the water flow direction moves back and forth, the original soil consolidation near anode is destoried by polarity reversal, water flow direction changes, so the polarity reversal treatment consumes more energy.

(2) Drainage effect is not obvious

Drainage effect of polarity reversal is impefect, the water content of anode and cathode does not change significantly. Due to the lower efficiency of polarity reversal, the dranage effect in anode and cathode are not obvious than usual electro-osmotic consolidation during test, water molecues in the soil under the action of current mostly concentrate between two electrodes, and failed to get effective discharge.

\section{Alternating current application}

Although reverse polarity can make the uniform soil consolidation, but the disadvantage of this method still cannot be ignored, especially for practical engineering application, long drainage duration, 
low efficiency, high energy consumption and cost, which brings great impact on engineering construction and investment. Also the experiment use alternating current(AC) instead of direct current(DC) during test, its action principle is roughly same with polarity reversal, soil also can get uniform consolidation.

Use AC power supply instead of DC, electro-osmotic dewatering under the condition of AC is effectively mainly in the region of frequency around $1 \mathrm{HZ}$. If polarity reverse too fast, frequency higher than 1HZ, the flow rate and the drainage effiency will be decreased. The principle of AC applied in the consolidation drainage is similar with polarity reversal treatment, the engery consumption under $\mathrm{AC}$ is much higher than under DC, and efficiency is lower than under DC.

\section{Inject chemical solution}

Due to a large amount of potential lost at the soil-electrode contacts during electro-osmotic consolidation of soil, Interface resistance is increased, and a lot of potential can not be effectivly used at the beginning of the test, only $35 \%$ of the gradient can effectively applied to electrode. With the electro-osmotic effects last longer, more seriously corrosion at the two ends of electrode, electro-osmotic consolidation efficiency is decreased faster. For the purpose of improve performance, solve problem of electrode erossion, insert chemecial solution during electro-osmotic consolidation.

Add chemecal solution to electro-osmotic consolidation, for example: Nacl, $\mathrm{Cacl}_{2}$ chemecial solution $^{[3]}$, through chemical reaction exchange Ions to alter the characteristics of soil particles, $k_{e}$ value is increased with drainage and consolidation rate, It becomes twice the initial state, and with the decrease of soil water content, soil shear strength is increased and the void ratio is decreased. Under the same concentration of $\mathrm{Cacl}_{2}$ and $\mathrm{Nacl}$ solution, former electro-osmotic consolidation efficiency is better than the latter, since $k_{e}$ value of test soil that inject $\mathrm{Cacl}_{2}$ solution is bigger than that inject Nacl solution, which can achieve better effect for consolidation. Chemical solution made initial potential loss at electrode from $35 \%$ decrese to $10 \%$. Water flow has no direct relationship with chemecial consentration, under the action of electro-osmotic consolidation, electro-osmotic effiency is improved with increase of $k_{e}$ value.

Chemical solution to improve the electro-osmotic effiency still at the experiment stage, this method is not widely used in the practical engineering, since the solution reaction with soil may cause a series other environment affects, especially for contaminted soil which may containes unknown chemical components, the characteristics of soil may change, so the limition of this method still exists, and needs further more study and research.

\section{Electro-osmosis combine with other consolidation methods}

Simply use electro-osmotic consolidation method to improve soft clay still has a lot of drawbacks, better effect can be reached by combine with other methods, since the high energy consumption for simple electro-osmotic consolidation, and from economical aspects, electro-osmotic consolidation method is mainly used for discharge weakly bound water. Vacuum preloading workes with electro-osmotic consolidation method is suitable for larger plasticity index clay and silty clay, especially for the soil with higher initial water content ${ }^{[2]}$. At first, Use vacuum preloading method to discharge large amount of free water from the soil with high initial water content, then at the later stage of improvement, use electro-osmosis method to discharge the weakly bound water that is difficult to discharge by vacuum preloading method. Meanwhile, vacuum preloading method can eliminate the oxygen and hydrogen near electrode, reduce interface resistance, improve soil strength and electro-osmotic efficiency.

In recent years, new conductive material EKG has been applied in many place, EKG material overcame the corrosion problem brought by traditional metal electrode, it can be used as both anode and cathode which makes electrode converse easily, application of EKG material is a new breakthrough in use of electrode. Meanwhile, EKG as electrode material has good effects, soil water content is decreased significantly, shear strength is greatly enhanced as shown in the Fig. $3^{[5]}$. 


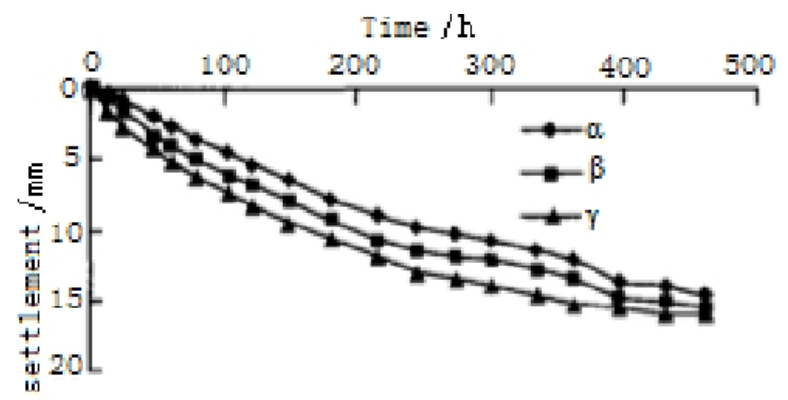

Fig. 3 Consolidation curve of electroosmosis

Using EKG material as electrodes still has problem of high resistivity of conductive plastic wire, and large engery consumption ${ }^{[5]}$, but it is a promising material for improve electro-osmotic effiency in the future.

\section{Conclusions}

(1) Electro-osmotic consolidation is used as a new method to treat soft soil, although it has better applicability than gravity consolidation method in dealing with low permeability saturated clay, its inherent defects brought by improvement principle needs further research and study.

(2) Polarity reversal method can solve uneven settlement problem of the electro-osmotic consolidation, but it exists defects such as large energy consumption, low efficiency, drainage effect is not obvious. The relationship between PH and PI value changes with electro-osmotic consolidation efficiency needs more tests.

(3) Principle and defects of apply AC during electro-osmotic consolidation is similar with polarity reversal method, the best electro-osmotic consolidation effect can be achieved under alternating frequency of $1 \mathrm{~Hz}$.

(4) Inject saline solution can effectively improve consolidation effeciency though increase $k_{e}$ value, and reduce soil-electrode interface resistance, but application is limited because of the complex soil characteristics may cause other chemical reaction.

(5) Vacuum preloading combine with electro-osmotic consolidation method is applied to consolidate soil, and the application of EKG new material has promising future, but large engery comsupmtion is a major problem that needs to be concerned in the practical engineering field.

\section{References}

[1] HU Li-ming, WU Wei-ling, WU Hui. Theoretical analysis and numerical simulation of electroosmosis consolidation for soft clay[J], Rock and soil mechanic, 2010,31(12):3977-3983.

[2] CAO Yong-hua,GAO Zhi-yi. Some Problems on Theory and Application of Drainage Consolidation in Electroosmotic for Ground Improvement[J], China harbour engineering, 2010(3):23-25.

[3] Shao-Chi Chien, Chang-Yu Ou, Ming-Kuang Wang. Injection of saline solutions to improve the electro-osmotic pressure and consolidation of foundation soil[J], Applied clay science, 44(2009):218-224.

[4] G.Lefebvre, T.V. Pavate. A study on Electro-osmotic consolidation of soft clays[J], Third international conference on case histories in geotechnical engineering, 1993, paper No.7.44:1131-1137.

[5] HU Yu-chen, WA NG Zhao, ZHUANG Yan-feng. Experimental studies on electro-osmotic consolidation of soft clay using EKG electrodes[J], Chinese journal of geotechnical engineering, $2005,27(5): 582-586$. 